\title{
Salivary alpha-amylase activity-An indicator of relaxation response in perioperative patients ${ }^{*}$
}

\author{
Chika Minowa $^{1 \#}$, Kikuyo Koitabashi ${ }^{2}$ \\ ${ }^{1}$ Department of Nursing, Saku University, Saku, Japan \\ ${ }^{2}$ Graduate School of Health Science, Gunma University, Maebashi, Japan \\ Email: "
}

Received 23 July 2012; revised 20 August 2012; accepted 31 August 2012

\begin{abstract}
The psychological stress of most perioperative patients has been reduced by many interventions. In order to evaluate the effects of these interventions, biomarkers are being considered as objective assessment tools in addition to the subjective assessment tools. It has been thought that salivary $\alpha$-amylase (sAA) activity is associated with sympathetic nervous system activity, which reflects psychological stress. We examined the relationship between sAA, self-reported data, and autonomic nervous system activity to assess the reliability of sAA as a marker for relaxing response in surgery-related stress. The sAA, the State anxiety, Visual Analogue Scale (VAS) of pain, and heart rate variability were used to assess prior to and after the relaxation on the day before surgery, and on postoperative days (POD) 1, 2, 3, and 7. A total of 75 patients participated and 301 relaxing response readings were obtained. We found that the sAA significantly decreased depending upon the pain and anxiety by relaxation, but they were unrelated to sympathetic nervous system activity. There was weak correlation between the change in sAA, and anxiety and VAS pain. Thus, sAA appears to be an easy-to-use, non-invasive and good marker for relaxing response within a short period in surgery-related stress patients.
\end{abstract}

Keywords: Anxiety; Pain; Salivary $\alpha$-Amylase Activity; Autonomic Nervous System; Surgical Stress

\section{INTRODUCTION}

The psychological stress that most perioperative patients feel has been reduced by many interventions, including providing information [1,2], relaxation [3,4], hypnosis [5], and massage therapy [6]. In order to evaluate the

${ }^{*}$ Conflicts of Interest: None declared.

${ }^{\#}$ Corresponding author. effects of these interventions, subjective assessment tools such as patient self-reporting have been utilized to measure the relaxation response. However, when patients do not reliably report, these subjective assessment tools have limited and disputable value. Given these limitations, biomarkers are being considered as objective assessment tools. Since the act of drawing blood itself induces stress, it cannot be used to reliably measure a stress response. Instead, salivary biomarkers, which can be sampled noninvasively, have been evaluated as stress markers. There are many advantages to using salivary biomarkers, including the rapid appearance of proteins in the saliva in response to stimuli, and the ability to easily measure salivary $\alpha$-amylase (sAA) at a patient's bedside using a portable measuring instrument [7]. An increase in stress level increases sympathetic activity and decreases parasympathetic activity [8]. This results in secretion of sAA because of the activation of the autonomic nervous system, which controls the activity of the salivary glands $[9,10]$.

Some studies have indicated that sAA increase during the psychological stress of skydiving [11], memory test and viewing a stressful video [12], playing a stressful video game [13], the noise of nurses working in an intensive care unit [14], Trier Social Stress Test [15], mental arithmetic stress [16] and viewing a stressful video [17]. sAA might be a good indicator for psychological stress and can be a useful assessment tool to evaluate the effects of nursing interventions for patients under stress. Some studies have already used sAA as an index to evaluate treatments such as epidural block in chronic pain [18], reflexology in dementia [19], and exposure to natural sound while undergoing epidural anesthesia surgery [20]. However, there are only few reports evaluating the relationship between sAA and relaxation response in patients who experience surgery-related stress. The aim of this current study is to investigate the relationships between sAA, self-reported pain and anxiety, and autonomic nervous system function in perioperative patients. 


\section{METHODS}

\subsection{Participants}

Patients were recruited from the Asama General Hospital and Saku Central Hospital between March 2009 and March 2012 in this prospective experimental study. Enrollment eligibility included: 1) scheduled breast cancer surgery under general anesthesia; 2) age of $\geq 20 \mathrm{yrs} ; 3$ ) female; 4) not having metastasis; 5) first cancer surgery; 6) ability to read, hear, write, and speak Japanese to answer the questionnaire questions. Breast cancer patients were enrolled because they already have high anxiety before surgery, and their anxiety remains high even after the surgery $[21,22]$. Precisely, it is estimated that they are in high stress condition during the measurement period.

\subsection{Study Protocol}

Participants were informed of the study purpose, procedures, and risks and benefits, as well as that collected data did not include identifying information, and that they could withdraw at any time without influencing medical treatment or nursing care. The study protocol was approved by the Saku University Ethics Committee, the Asama General Hospital Ethics Committee, and the Saku Central Hospital Ethics Committee. All participants signed an informed consent form.

Participants were evaluated the day before operation, and on postoperative days (POD) 1, 2, 3, and 7 during their hospital stay. All participants were evaluated for prior to and after the relaxation of $10-20$ min between 13:00 and 18:00 hrs to minimize circadian rhythm variability [23]. Relaxation consisted of rest with their eyes closed or listening to $\mathrm{CD}$. At least $1 \mathrm{~h}$ before their evaluation, participants were required to refrain from eating and brushing their teeth, but they could take their pain medication.

\subsection{Measurements}

Demographic and clinical data were collected from the electronic medical chart.

\subsubsection{Salivary Alpha Amylase (sAA)}

sAA was measured using a portable salivary amylase monitor (Nipro Co., Japan). Saliva was collected by a test strip placed under the tongue for approximately $30 \mathrm{~s}$, and immediately measured; the result was displayed in $60 \mathrm{~s}$. The salivary amylase monitor used in this study has been thoroughly examined and assessed to be valid and reliable [24-26]. Stress level was considered high when the value was $\geq 40 \mathrm{kU} / \mathrm{L}$. The coefficient of variation was less than $9 \%$ [7]. If an error was displayed twice due to less saliva after surgery or the patient could not place the test strip placed in the mouth because of nausea, the test was aborted to avoid any further stress on the patient.

\subsubsection{State-Trait Anxiety Inventory (STAI)}

Anxiety is the biggest factor of stress in breast cancer patients, and it has been known to reduce as a response to relaxation. Spielberger et al. defined STAI-s as the current emotional state that exists at the time, while trait anxiety (STAI-t) is a personal characteristic which does not change depending upon the situation. The STAI-s questionnaire involves a 20 -item scale based upon selfreporting measures and typically requires a very short time to complete. Items are rated on a 4-point scale, with scores ranging from 20 to 80 , with 80 indicating high anxiety [27]. This scale is widely used and is considered to be valid and reliable. We used STAI translated into Japanese, which is also considered valid and reliable [28]. Researchers evaluated the questionnaire according to the manual of the questionnaire STAI.

\subsubsection{Visual Analogue Scale of Pain (VAS Pain)}

Pain is one of the significant factors of stress in breast surgery patients, and pain is reduced by breaking of the pain-anxiety-tension cycle by relaxation.

This scale consisted of a $100 \mathrm{~mm}$ horizontal line with the phrases "no pain at all" on the left end of the line and "the worst pain I have ever felt" on the right end of the line. Participants indicated their current level of pain by marking on the horizontal line $[29,30]$.

\subsubsection{Heart Rate Variability (HRV)}

sAA has been proposed as a measure of sympathetic nervous system (SNS) activity. Psychological stress can induce a "fight or flight" response, resulting in a rise in the activation of sympathetic adrenal modular system $[15,31]$. HRV is determined by measuring the change over time of cardiac beats per minute; the change in power spectra is analyzed and translated into a frequency component, and is then used as an index of the activity of heart autonomic nervous system [32]. High frequency (HF: $0.15-0.4 \mathrm{~Hz}$ ) is thought to reflect parasympathetic nervous system activity while low frequency (LF: 0.04 $0.15 \mathrm{~Hz}$ ) is thought to reflect both parasympathetic and sympathetic nervous system activity. The low-to-high frequency ratio $(\mathrm{LF} / \mathrm{HF})$ is thought to reflect SNS activity. Electrocardiograph was recorded with LRR-03 (GMS Co., Tokyo, Japan) and the data was transferred to computer, where a software was used to calculate heart rate, LF and HF values, and the LF/HF of HRV by the maximum entropy method (MemCalc/Tarawa, Suwa Trust, Tokyo, Japan). Although parasympathetic nervous system activity was influenced by breathing, we did not perform a control, but no patients had respiratory rate of $\leq 10$ breathing/min during measurement. 


\subsubsection{Systolic Blood Pressure}

Systolic blood pressure was measured using an automated sphygmomanometer on the upper arm furthest from the surgical site.

\subsection{Statistical Analysis}

All data were tested for normality using the Kolmogorov-Smirnov test prior to analysis. Non-parametric analysis was carried out to test for the association between sAA and the subjective and objective data using Spearman's correlation test. They were presented as the median $[25,75 \%$ IQR], and we used Wilcoxon's signedrank test to determine significant differences between data collected prior to and after relaxation. Parametric data were presented as mean \pm standard deviation, and Student's t-test was used to determine significant differences between data collected prior to and after relaxation.

All analyses were performed using SPSS Advanced Statistics $18 ; \mathrm{p}<0.05$ was considered significant.

\section{RESULTS}

\subsection{Sample Characteristics}

Seventy-five patients participated in the study and 301 relaxation response data were obtained (the day before operation, $\mathrm{n}=71$; POD $1, \mathrm{n}=60$; POD2, $\mathrm{n}=65$; POD3, $\mathrm{n}=62 ;$ POD7, $\mathrm{n}=43$ ). Table 1 shows demographic and medical characteristics of participants. None of the patients were in stage 0 of breast cancer; $43(57.3 \%)$ patients were in stage I, which was the most frequent stage; $21(28.0 \%)$ in stage II; $9(12.0 \%)$ in stage III; and 2 $(2.7 \%)$ in stage IV, which was the least frequent stage.

The most common surgical procedure was breastconserving surgery $(\mathrm{n}=36,48.0 \%)$, followed by mastectomy $(\mathrm{n}=27,36.0 \%)$, and immediate reconstruction following mastectomy $(\mathrm{n}=12,16.0 \%)$.

\subsection{Association between SAA, STAI-s, and VAS Pain}

Table 2 shows the association of the sAA, STAI-s, and VAS pain. The sAA was high $(54.0 \mathrm{ku} / \mathrm{l})$ the day before operation and also on POD7 (44.0 ku/l) prior to relaxation. This indicates that the patients were in stress during the perioperative period. The sAA was found to be significantly different between pre-test and post-test on all days $(p<0.05)$, except POD1 $(p=0.367)$ and were reflected by STAI-s the day before operation, POD1 and POD3 $(p<0.05)$ or VAS pain reflected by POD1, POD2 and POD7 $(\mathrm{p}<0.05)$. The sAA significantly decreased $(p<0.05)$ on significant decrease $(p<0.05)$ in either STAI-s or pain.

The change in sAA did not show significant correlation with the changes in STAI-s $(r=-0.134-0.267)$ and the change in VAS pain $(r=-0.002-0.231)$.

\subsection{Association between sAA and the Autonomic Nervous System Activity}

Table 3 shows the association of the sAA and autonomic nervous system activity. The autonomic nervous system activity measured by pre-post test of heart rate $(\mathrm{p}$ $=0.025-0.485)$, systolic blood pressure $(\mathrm{p}=0.016-$ $0.660)$, and HF $(p=0.013-0.191)$, partially reflected sAA, though LF/HF ( $p=0.233-0.812)$ did not reflect sAA.

There was no significant relationship between percent change in sAA and percent change in heart rate $(\mathrm{r}=$ $-0.021-0.232$ ), percent change in systolic blood pressure $(r=-0.073-0.129)$, percent change in HF $(r=$ $-0.114-0.192)$, and mean difference in LF/HF $(r=$ $-0.037-0.075)$.

\section{DISCUSSION}

The aim of this study was to investigate the application of sAA in assessing the relaxation response in perioperative patients. We demonstrated a significant association between sAA and relaxation response depending on STAI-s and VAS pain. Our results are consistent with some of the previous studies. Hodgson \& Andersen [19] reported that sAA was associated with decrease in pain intensity under reflexology treatment for 4 weeks in patients with dementia. Takai et al. [17] also showed decreased sAA associated with STAI after viewing soothing video. In addition, Arai et al. [20] reported that intraoperative natural sound decreased sAA in patients undergoing inguinal hernia under epidural anesthesia. Similar

Table 1. Demographic and medical characteristics.

\begin{tabular}{lcc}
\hline \multicolumn{1}{c}{ Variable } & mean \pm SD & range \\
\hline Age (years) & $55.5 \pm 11.2$ & $32-77$ \\
Body mass index & $22.5 \pm 3.7$ & $15.7-32.1$ \\
Duration of operation & $193.8 \pm 120.4$ & $37-527$ \\
Trait-anxiety score & $43.6 \pm 11.2$ & $20-72$ \\
\hline & $\mathrm{n}$ & $\%$
\end{tabular}

Stage of breast cancer

$\begin{array}{lcc}\text { Stage } 0 & 0 & 0 \\ \text { Stage I } & 43 & 57.3 \\ \text { Stage II } & 21 & 28.0 \\ \text { Stage III } & 9 & 12.0 \\ \text { Stage IV } & 2 & 2.7\end{array}$

Surgical procedure

Breast-conserving surgery $\quad 36 \quad 48.0$

$\begin{array}{lll}\text { Mastectomy } & 27 & 36.0\end{array}$

Mastectomy \& immediate reconstruction $\quad 12 \quad 16.0$ 


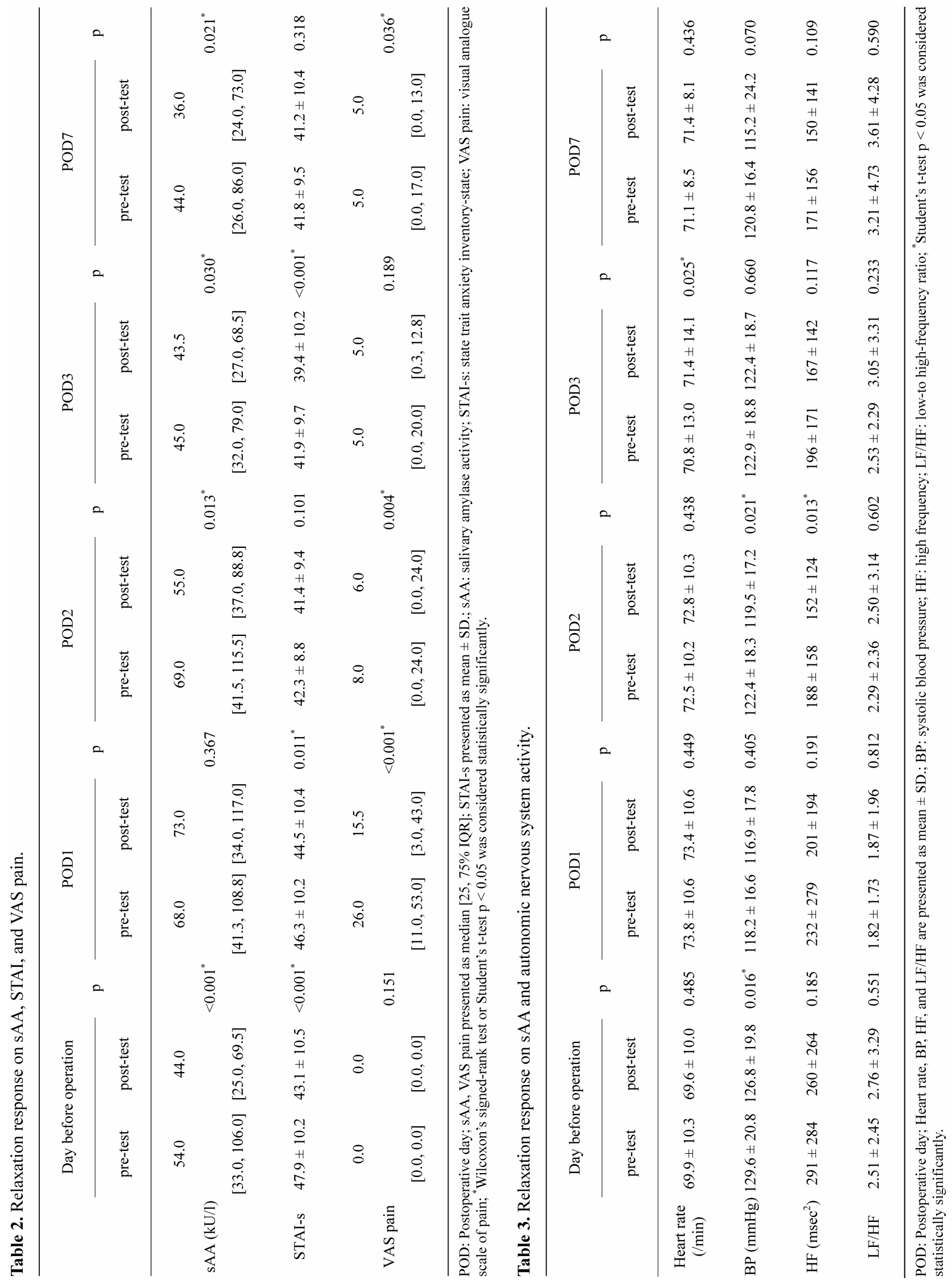


to previous studies, this study showed that sAA is decreased by relaxation depending on the objective response.

Previous studies have shown the correlation between sAA and STAI. Noto et al. [16] found a correlation between sAA and STAI $(r=0.589)$ within a short time prior to and after exposing the young healthy volunteers in a laboratory to mental arithmetic stress. Takai et al. [17] used a stressful video to induced stress and showed direct correlation between sAA and STAI. In both studies, participants were young healthy volunteers, the participants were subjected to soft stress, and the change in sAA was compared within short time. Moreover, the studies were carried out under controlled special conditions, which differ from the clinical setting. Therefore, it is thought that SAA and STAI had a weak correlation in this study.

The correlation of SAA and pain was shown by Shirasaki et al. [18], who evaluated the relationship between pain intensity and SAA in patients with chronic lower back pain. Surgical patients were used as a control group. VAS pain levels and sAA were significantly decreased after an epidural block was used as an anesthetic, and they showed significant correlation $(\mathrm{r}=0.561)$. However, most of the studies found small correlation between sAA and pain like our study. Arai et al. reported that SAA and VAS pain levels had a positive, but small correlation in patients with cancer [33], while Campos et al. reported that there was no correlation between sAA and VAS pain levels in orthodontic patients [34]. Yet another study reported a small association between psychological scores and sAA in pregnancy wastage [35]. These reports indicate that sAA correlated with self-reported pain scores of patients with chronic pain and those subjected to soft stress, which were performed in a laboratory within a short time. However, a significant relationship is not reported in a clinical setting, which can be useful for patients experiencing postoperative pain.

Our results show that secretion of sAA is not associated with SNS activity. Even though there was pain stress and sAA had increased, LF/HF decreased on POD1 from the day before surgery. Some studies found that both LF/HF and HF decreased after surgery [36-38], while other studies failed to identify relationship between SNS activity and pain [39-41]. Our study is consistent with earlier studies $[9,42]$ that demonstrated that there were no or weak correlations between sAA and heart rate parameters. The association of secretion of sAA and SNS activity is still not clear.

The SAA is secreted from the parotid and is clearly a sympathetic activity [43]. Simultaneously, the amount of saliva produced is adjusted, and the parasympathetic nerves stimulate the secretion of saliva containing high levels of sAA, indicating that both branches of the auto- nomic nervous system participate in saliva secretion [10]. HRV, however, reflects heart autonomic nervous system activity, and sAA reflects the local autonomic nervous system activity of the salivary glands, which are mutually exclusive. There may be an influence of anesthesia and analgesic on the concentration of SAA especially on the POD1. This complicated mechanism of secretion of sAA is one the factors that sAA level is not significantly difference.

In this study, sAA always significantly decreased on significant decrease in either STAI or pain $(\mathrm{p}<0.05)$ after relaxation. This indicates that sAA may be a sensitive marker. sAA is affected by various things; hence, it is believed that the change in SAA may be much higher than the change in the numbers representing STAI and VAS. As, stress and relaxation affect sAA immediately in a clinical situation, previous studies as well as this study failed to find correlation between sAA and subjecttive data.

There are some limitations to this study. Participants were only women who had undergone breast cancer surgery; therefore, the result cannot be applied to general surgery patients.

Previously, measurement of sAA required an analyticcal instrument in a laboratory to perform an enzymelinked immunosorbent assay (ELISA) and saliva samples were preserved by freezing. For this study, a portable apparatus was used to measure sAA at the patient's bedside within 1 - 2 min. Since measurements can be quickly obtained, results can be used in various ways in a clinical setting to improve the treatment of patients.

Future research is should consider larger sample size, patients who have undergone general surgery such as pulmonary or abdominal surgery, sex difference, and comparison of sAA with other stress markers.

\section{CONCLUSION}

Our findings suggest that the sAA significantly decreased by relaxation intervention in perioperative patients, but they were unrelated to sympathetic nervous system activity. Thus, assessing sAA can be an easy to use, non-invasive, and good marker for relaxation response in patients with surgery-related stress in clinical settings and nursing research

\section{ACKNOWLEDGEMENTS}

We wish to thank all of the patients who participated, Dr. H. Ishige (Saku Central Hospital), Dr. H. Masuda (Masuda Clinic), and the breast surgeon team of Asama General Hospital.

\section{REFERENCES}

[1] Leinonen, T. and Leino-Kilpi, H. (1999) Research in 
peri-operative nursing care. Journal of Clinical Nursing, 8, 123-138. doi:10.1046/j.1365-2702.1999.00239.x

[2] Johansson, K., Nuutila, L., Virtanen, H., Katajisto, J. and Salanterä, S. (2005) Preoperative education for orthopaedic patients: Systematic review. Journal of Advanced Nursing, 50, 212-223. doi:10.1111/j.1365-2648.2005.03381.x

[3] Roykulcharoen, V. and Good, M. (2004) Systematic relaxation to relieve postoperative pain. Journal of $A d-$ vanced Nursing, 48, 140-148. doi:10.1111/j.1365-2648.2004.03181.x

[4] Seers, K. and Carroll, D. (1998) Relaxation techniques for acute pain management: A systematic review. Journal of Advanced Nursing, 27, 466-475. doi:10.1046/j.1365-2648.1998.00538.x

[5] Montgomery, G.H., Bovbjerg, D.H., Schnur, J.B., David, D., Goldfarb, A., Weltz, C.R., Schechter, C., Graff-Zivin, J., Tatrow, K., Price, D.D. and Silverstein, J.H. (2007) A randomized clinical trial of a brief hypnosis intervention to control side effects in breast surgery patients. Journal of the National Cancer Institute, 99, 1304-1312. doi:10.1093/jnci/djm106

[6] Bauer, B.A., Cutshall, S.M., Wentworth, L.J., Engen, D., Messner, P.K., Wood, C.M., Brekke, K.M., Kelly, R.F. and Sundt, T.M. (2010) Effect of massage therapy on pain, anxiety, and tension after cardiac surgery: A randomized study. Complementary Therapies in Clinical Practice, 16, 70-75. doi:10.1016/j.ctcp.2009.06.012

[7] Yamaguchi, M., Kanemaru, M., Kanemori, T. and Mizuno, Y. (2003) Flow-injection-type biosensor system for salivary amylase activity. Biosensors \& Bioelectronics, 18, 835-840. doi:10.1016/S0956-5663(03)00007-1

[8] Benson, H. and Klipper, M.Z. (2001) The relaxation response. Harper Collins Publishers Inc., New York.

[9] Nater, U.M. and Rohleder, N. (2009) Salivary alpha-amylase as a non-invasive biomarker for the sympathetic nervous system: Current state of research. Psychoneuroendocrinology, 34, 486-496. doi:10.1016/j.psyneuen.2009.01.014

[10] Proctor, G.B. and Carpenter, G.H. (2007) Regulation of salivary gland function by autonomic nerves. Autonomic Neuroscience, 133, 3-18. doi:10.1016/j.autneu.2006.10.006

[11] Chatterton, R.T. Jr., Vogelsong, K.M., Lu, Y.C. and Hudgens, G.A. (1997) Hormonal responses to psychological stress in men preparing for skydiving. The Journal of Clinical Endocrinology and Metabolism, 82, 2503-2509. doi:10.1210/jc.82.8.2503

[12] Bosch, J.A., De Geus, E.J.C., Veerman, E.C.I., Hoogstraten, J. and Amerongen, A.V.N. (2003) Innate secretory immunity in response to laboratory stressors that evoke distinct patterns of cardiac autonomic activity. Psychosomatic Medicine, 65, 245-258 doi:10.1097/01.PSY.0000058376.50240.2D

[13] Skosnik, P.D., Chatterton, R.T. Jr., Swisher, T. and Park, S. (2000) Modulation of attentional inhibition by norepinephrine and cortisol after psychological stress. International Journal of Psychophysiology, 36, 59-68. doi:10.1016/S0167-8760(99)00100-2
[14] Morrison, W.E., Haas, E.C., Shaffner, D.H., Garrett, E.S. and Fackler, J.C. (2003) Noise, stress, and annoyance in a pediatric intensive care unit. Critical Care Medicine, 31, 113-119. doi:10.1097/00003246-200301000-00018

[15] Nater, U.M., Rohleder, N., Gaab, J., Bergera, S., Juda, A., Kirschbaum, C. and Ehlert, U. (2005) Human salivary $\alpha$-amylase reactivity in a psychosocial stress paradigm. International Journal of Psychophysiology, 55, 333-342. doi:10.1016/j.ijpsycho.2004.09.009

[16] Noto, Y., Sato, T., Kudo, M., Kurata, K. and Hirota, K. (2005) The relationship between salivary biomarkers and state-trait anxiety inventory score under mental arithmetic stress: A pilot study. Anesthesia and Analgesia, 101, 1873-1876.doi:10.1213/01.ANE.0000184196.60838.8D

[17] Takai, N., Yamaguchi, Y., Aragakia, T., Eto, K., Uchihashia, K. and Nishikawa, Y. (2004) Effect of psychological stress on the salivary cortisol and amylase levels in healthy young adults. Archives of Oral Biology, 49, 963-968. doi:10.1016/j.archoralbio.2004.06.007

[18] Shirasaki, S., Fujii, H., Takahashi, M., Sato, T., Ebina, M., Noto, Y. and Hirota, K. (2007) Correlation between salivary $\alpha$-amylase activity and pain scale in patients with chronic pain. Regional Anesthesia and Pain Medicine, 32, 120-123. doi:10.1016/j.rapm.2006.11.008

[19] Hodgson, N.A. and Andersen, S. (2008) The clinical efficacy of reflexology in nursing home residents with dementia. Journal of Alternative and Complementary Medicine, 14, 269-275. doi:10.1089/acm.2007.0577

[20] Arai, Y.-C.P., Sakakibara, S., Ito A., Ohshima, K., Sakakibara, T., Nishi, T., Hibino, S., Niwa, S. and Kuniyoshi, K. (2008) Intra-operative natural sound decreases salivary amylase activity of patients undergoing inguinal hernia repair under epidural anesthesia. Acta Anaesthesiologica Scandinavica, 52, 987-990. doi:10.1111/j.1399-6576.2008.01649.x

[21] Den Oudsten, B.L., Van Heck, G.L., Van der Steeg, A.F.W., Roukema, J.A. and De Vries, J. (2009) Predictors of depressive symptoms 12 months after surgical treatment of early-stage breast cancer. Psycho-Oncology, 18, 1230-1237. doi: $10.1002 /$ pon. 1518

[22] Li, X.-M., Zhou, K.-N., Yan, H., Wang, D.-L. and Zhang, Y.-P. (2012) Effects of music therapy on anxiety of patients with breast cancer after radical mastectomy: A randomized clinical trial. Journal of Advanced Nursing, 68, 1145-1155. doi:10.1111/j.1365-2648.2011.05824.x

[23] Nater, U.M., Rohleder, N., Schlotz, W., Ehlert, U. and Kirschbaum, C. (2007) Determinants of the diurnal course of salivary alpha-amylase. Psychoneuroendocrinology, 32, 392-401. doi:10.1016/i.psyneuen.2007.02.007

[24] Yamaguchi, M., Kanemori, T., Kanemaru, M., Takai, N., Mizuno, Y. and Yoshida, H. (2004) Performance evaluation of salivary amylase activity monitor. Biosensors \& Bioelectronics, 20, 491-497. doi:10.1016/j.bios.2004.02.012

[25] Yamaguchi, M., Deguchi, M., Wakasugi, J., Ono, S., Takai, N., Higashi, T. and Mizuno, Y. (2006) Hand-held monitor of sympathetic nervous system using salivary amylase activity and its validation by driver fatigue assessment. Biosensors \& Bioelectronics, 21, 1007-1014. 
doi:10.1016/j.bios.2005.03.014

[26] Shetty, V., Zigler, C., Robles, T.F., Elashoff, D. and Yamaguchi, M. (2011) Developmental validation of a pointof-care, salivary $\alpha$-amylase biosensor. Psychoneuroendocrinology, 36, 193-199. doi:10.1016/j.psyneuen.2010.07.008

[27] Spielberger, C.D., Gorsuch, R.L., Lushene, P.R., Vagg, P.R. and Jacobs, G.A. (1983) Manual for the state-trait anxiety inventory (form Y) ("self-evaluation questionnaire”). Consulting Psychologists Press Inc., Palo Alto.

[28] Hidano, T., Fukuhara, M., Iwawaki, S., Soga, S. and Spielberger, C.D. (2000) New STAI manual for the state-trait anxiety inventory-form JYZ. Jitsumu Kyouiku Press Inc., Tokyo.

[29] Kelly, A.-M. (1998) Does the clinically significant difference in visual analog scale pain scores vary with gender, age, or cause of pain? Academic Emergency Medicine, 5, 1086-1090. doi:10.1111/j.1553-2712.1998.tb02667.x

[30] Bijur, P.E., Silver, W. and Gallagher, E.J. (2001) Reliability of the visual analog scale for measurement of acute pain. Academic Emergency Medicine, 8, 1153-1157. doi:10.1111/j.1553-2712.2001.tb01132.x

[31] Rohleder, N., Nater, U.M., Wolf, J.M., Ehlert, U. and Kirschbaum, C. (2004) Psychosocial stress-induced activation of salivary alpha-amylase: An indicator of sympathetic activity? Annals of the New York Academy of Sciences, 1032, 258-263. doi:10.1196/annals.1314.033

[32] Akselrod, S., Gordon, D., Ubel, F.A., Shannon, D.C., Berger, A.C. and Cohen, R.J. (1981) Power spectrum analysis of heart rate fluctuation: A quantitative probe of beat-to-beat cardiovascular control. Science, 213, 220222. doi:10.1126/science. 6166045

[33] Arai, Y.-C.P., Matsubara, T., Shimo, K., Osuga, T., Shida, T., Suzuki, C., Ohta, A., Tohyama, Y., Nishida, K. and Arakawa, M. (2009) Small correlation between salivary $\alpha$-amylase activity and intensity in patients with cancer pain. Acta Anaesthesiologica Scandinavica, 53, 408. doi:10.1111/j.1399-6576.2008.01833.x

[34] Campos, M.J., Raposo, N.R., Ferreira, A.P. and Vitral, R.W. (2011) Salivary $\alpha$-amylase activity: A possible indicator of pain-induced stress in orthodontic patients. Pain Medicine, 12, 1162-1166. doi:10.1111/j.1526-4637.2011.01185.x
[35] Milad, M.P., Klock, S.C., Moses, S. and Chatterton, R. (1998) Stress and anxiety do not result in pregnancy wastage. Human Reproduction, 13, 2296-2300. doi:10.1093/humrep/13.8.2296

[36] Amar, D., Fleisher, M., Pantuck, C.B., Shamoon, H., Zhang, H., Roistacher, N., Leung, D.H.Y., Ginsburg, I. and Smiley, R.M. (1998) Persistent alterations of the autonomic nervous system after noncardiac surgery. $A n$ esthesiology, 89, 30-42. doi:10.1097/00000542-199807000-00008

[37] Marsch, S.C.U., Skarvan, K., Schaefer, H.-G., Naegeli, B., Paganoni, R., Castelli, I. and Scheidegger, D. (1994) Prolonged decrease in heart rate variability after elective hip arthoplasty. British Journal of Anaesthesia, 72, 643649. doi:10.1093/bja/72.6.643

[38] Ushiyama, T., Mizushige, K., Wakabayashi, H., Nakatsu, T., Ishimura, K., Tsuboi, Y., Maeta, H. and Suzuki, Y. (2008) Analysis of heart rate variability as an index of noncardiac surgical stress. Heart and Vessels, 23, 53-59. doi:10.1007/s00380-007-0997-6

[39] Ledowski, T., Reimer, M., Chavez, V., Kapoor, V. and Wenk, M. (2012) Effects of acute postoperative pain on catecholamine plasma levels, hemodynamic parameters, and cardiac autonomic control. Pain, 153, 759-764. doi:10.1016/j.pain.2011.11.002

[40] Kang, Y. (2010) Psychological stress-induced changes in salivary $\alpha$-amylase and adrenergic activity. Nursing \& Health Sciences, 12, 477-484. doi:10.1111/j.1442-2018.2010.00562.x

[41] Ledowski, T., Ang, B., Schmarbeck, T. and Rhodes, J. (2009) Monitoring of sympathetic tone to assess postoperative pain: Skin conductance vs surgical stress index. Anaesthesia, 64, 727-731. doi:10.1111/j.1365-2044.2008.05834.x

[42] Rohleder, N., Wolf, J.M., Maldonando, E.F. and Kirschbaum, C. (2006) The psychosocial stress-induced increase in salivary alpha-amylase is independent of saliva flow rate. Psychophysiology, 43, 645-652. doi:10.111/j.1469-8986.2006.00457.x

[43] Bosch, J.A., Veerman, E.C.I., De Geus, E.J. and Proctor, G.B. (2011) $\alpha$-Amylase as a reliable and convenient measure of sympathetic activity: Don't start salivating just yet! Psychoneuroendocrinology, 36, 449-453. doi:10.1016/j.psyneuen.2010.12.019 